\title{
I liked the postcard you sent Abe and I: Context-sensitive coding of syntax and other procedural knowledge
}

\author{
WAYNE A. WICKELGREN \\ University of Oregon, Eugene, Oregon 97403
}

\begin{abstract}
A theory of the coding of syntactic knowledge is described and illustrated by means of its predictions regarding certain pronomial case errors in children. The theory assumes two levels of syntactic coding: (1) word classes (noun, verb, adjective, adverb, articles, pronouns, prepositions, conjunctions) and (2) concept functions in a proposition (relation, agent, object, recipient, location, etc.). Both word-class nodes and concept-function nodes are context-sensitive (overlapping triple) codes, which is the key to their encoding of serial order in both recognition and production.
\end{abstract}

\section{PRONOMIAL CASE ERRORS}

Yesterday my daughter Ingrid wrote a letter containing the common grammatical error expressed in the title of this paper. She does not make this pronoun case error with a single object (e.g., "I liked the postcard you sent me"). Many times I have heard children make this sort of pronoun case error for compound objects and subjects, when I knew they would not make this error for a single pronoun serving the same function in the sentence. This time, because of a theory I recently generated, I realized that the error was of some theoretical importance. So I did a bit of informal testing of my children, Ingrid and Abe, to provide some "facts" to illustrate the predictions of the theory. ${ }^{1}$

Further constraining the theoretical interpretation of this syntactic error is the fact that my children do not err on the pronoun case if they generate the compound object in the "impolite" order, which puts the first-person pronoun first in the compound noun phase (e.g., "I liked the postcard you sent me and Abe"). What these three linguistic facts appear to indicate is that the syntactic rule that determines objective pronoun case is originally very local and nonhierarchical in character. My children know to choose "me" over "I" when the first-person singular pronoun immediately follows a verb or preposition, regardless of whether the pronoun is used as the sole object or as the first element of a compound object. They do not determine the pronoun's case by knowing its function (use) in the sentence, because if they did they would not get the case wrong when the pronoun is the second element of a compound object (e.g., "to

This research was supported by Grant NIE-G-77-008, from the National Institute of Education. Requests for reprints should be sent to Wayne A. Wickelgren, Psychology Department, University of Oregon, Eugene, Oregon 97403.
Ingrid and I," or "to Ingrid and he," or "to Ingrid and they").

Nominative case for a compound subject shows the same error pattern. My children consistently choose nominative case for single pronoun subjects of sentences and correctly choose nominative case for the second element of a compound subject (e.g., "Abe and I sent a postcard to you," or "Abe and she went to the store"). They make mistakes on compound subjects only when the pronoun is the first element of the compound (e.g., "Me and Ingrid want to go to the movies," or "Him and Susie played Monolopy"). Note that the commonality in the error pattern is that the pronoun farthest from the case-governing relational term (verb or preposition) is the one that demonstrates case errors. The pronoun adjacent to the relational term is assigned proper case.

\section{CONTEXT-SENSITIVE CODING OF SYNTAX}

I took note of this phenomenon yesterday because it fits with a theory I generated a few weeks ago. The theory is concerned with the encoding of procedural knowledge, in this case, with the mental encoding of syntactic knowledge. The basic assumption of the theory is that the syntactic structures of sentences we generate and recognize are encoded by a set of context-sensitive syntactic concept nodes. The theory extends the idea of context-sensitive coding originally generated for the encoding of the order of phonetic segments in words (Wickelgren, 1969a, 1969b, 1972, 1976) to the syntactic level of language. For example, the title "sentence" might be encoded as shown in Figure 1.

At the lowest syntactic level of analysis, each word is associated to some syntactic class concept. Grammatical "function" words, such as articles, prepositions, conjunctions, and pronouns, are assumed to be classes with only one member word in adults and to be classes 


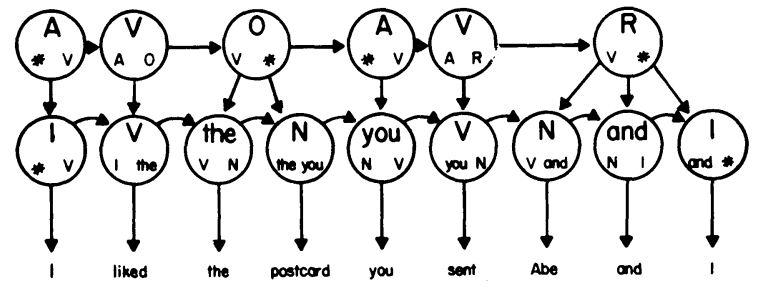

Figure 1. Two-level context-sensitive coding of the syntactic structure of the title sentence in terms of overlapping triples of syntactic class concepts. Note that the substantive noun (N), verb (V), adjective and adverb classes are assumed to be rather large classes, while the grammatical word classes-articles, pronouns, prepositions, and conjunctions-are assumed to be very small and specific classes. The lower level of syntactic coding is concerned with word classes, while the higher level is concerned with the semantic function of concepts in propositions.

with one or only a few members in children. As indicated in Figure 1, the "content" words, such as nouns, verbs, adjectives, and adverbs, may be encoded into only a few syntactic categories, each having a large number of members. Specifying exactly what these syntactic categories are for adults and children is beyond the scope of this paper. However, I imagine, for example, that nouns would have to be subdivided into categories to represent all existing combinations of the following features: mass vs. count, singular vs. plural, human vs. nonhuman, animate vs. inanimate, and a few others.

Syntactic development involves the acquisition, generalization, and differentiation of these grammatical classes. To give some indication of what is meant by this grand statement, imagine that a child's first twoword sentence is "Mama go." We assume the child acquires the syntactic concept nodes: \#mama go $_{\text {o }}$ and

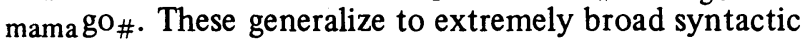
categories at some intermediate stage of development, possibly ${ }_{\#} \mathrm{~N}_{\mathrm{V}}$ and ${ }_{\mathrm{N}} \mathrm{V}_{\#}$, where $\mathrm{N}$ includes both nouns and pronouns and $\mathrm{V}$ includes all verbs. However, eventually, the ${ }_{\#} \mathrm{~N}_{\mathrm{V}}$ and ${ }_{\mathrm{N}} \mathrm{V}_{\#}$ categories differentiate to reflect the pronoun-noun, mass-count, singularplural, and so on, distinctions. Further specification of the time course and learning mechanisms of syntactic development are beyond the scope of this paper, but see Anderson (1976) for some useful ideas. What is asserted here is that, at any stage of development, syntactic competence can be described by a set of context-sensitive syntactic category concepts.

Of course, there is more to syntactic knowledge than a single level of word-class concepts. Syntactic knowledge is hierarchical. Above the level of word-class concepts are one or more levels of phrase-class concepts, such as verb phrases (verb plus all auxilliaries, adverb modifiers, infinitives, and complements) and case phrases (agent, object, recipient, location, etc.). The order of verb, agent, object, recipient, and so on, phrases in a sentence can also be represented by contextsensitive coding, as illustrated in Figure 1. Left out of Figure 1 are the concept nodes, which also link the agent, verb, object, recipient nodes to the word nodes illustrated at the bottom of Figure 1 .

One of the grand objectives in psycholinguistics is to use the same encoding of syntactic knowledge in both recognition and production of sentences. The contextsensitive theory of syntax permits this. In recognition, words can activate the appropriate context-sensitive syntactic word-class nodes, and concepts cued by words and phrases can activate the appropriate contextsensitive semantic-function nodes. Production presumably starts with activation of a set of concept and proposition nodes in semantic memory. Such nodes logically must include the semantic-function level of syntactic knowledge. The concept nodes and semanticfunction nodes then prime (partially activate) word nodes and syntactic word-class nodes. Once a complete set of nodes, such as that shown in Figure 1, is primed (partially activated), they can be fully activated in the correct sequential order by activating the $\# x_{y}$ nodes and letting the associations direct the activation of subsequent nodes. There are level-coordination-ofactivation (and many other) problems being ignored in this paper, but that is how it goes. You do not solve all theoretical problems at once.

\section{FUNCTION OF CONTEXT-SENSITIVE CODING}

Context-sensitive coding permits the representation of an ordered set (a procedure) by means of an unordered set of nodes (a knowledge structure in a network). For example, a visual word such as "stale" can be represented by the unordered set of contextsensitive nodes: ${ }_{\#} s_{t},{ }_{s} t_{a}, t_{1} a_{1},{ }_{a} l_{e},{ }_{1} e_{\#}$. Context-sensitive coding has nodes representing essentially overlapping triples of actions and/or states of the procedure considered as an ordered set of context-free elements. An unordered set of such context-free nodes is inadequate to represent the order of the elements, as Lasley (1951) pointed out for words.

For example, in reading, just recognizing the unordered set of letters, $s, t, a, l, e$, does not discriminate between the words "stale," "steal," and "slate," even assuming the knowledge of the letter constituents of familiar words stored in our associative memories, to say nothing of our capacity correctly to read the letter order in new "made-up" words such as "setal" or "tesal." It is a remarkable fact that knowledge of just the immediate left and right neighbors of every element in an ordered set is sufficient to reconstruct the correct ordering for almost all possible ordered sets of small to moderate size (Wickelgren, 1969a, 1969b, 1972, 1976). Context-sensitive letter coding allows us to perceive all the overlapping letter triples of a word in parallel in reading and still retain the information 
concerning the order of the letters in the word. It accomplishes this without having to use separate nodes for the same letter in every possible serial position in words.

As discussed in the previous section, context-sensitive coding can be used to represent syntactic knowledge. Such context-sensitive coding in terms of overlapping triples of syntactic categories provides a functional encoding of procedural knowledge that will permit parallel activation of all of the syntactic elements of a long phrase or sentence, in reading or speech recognition, while retaining all of the necessary order information. This is equally true of the planning phase of sentence generation in writing or speech production. If one primes such a context-sensitive syntactic plan with each syntactic node associated to a word or phrase, the syntactic plan will program the sentence-generation process to produce grammtical sentences. Note that such a context-sensitive coding of procedural syntactic knowledge does achieve the elusive objective of using the same encoding of syntax for both sentence recognition and production.

Context-sensitive coding of syntactic knowledge can be hierarchical. At a higher level, the ordering of entire phrases may be controlled by a plan such as \#Agent Verb, Agent Verb Object, Verb Object \# for an active sentence, or \#Object Verb, Object Verb Agent, Verb Agent \# for a passive sentence. Finally, branching and looping control plans for procedural knowledge can be achieved by context-sensitive coding. For example, consider the plan shown in Figure 2, consisting of an alternation of action nodes $(1,2$, etc.) and state nodes $(a, b, e t c$.$) .$ Such a plan begins by performing act- 1 as controlled by action node $\#_{a}$, then runs through the loop repeatedly until the environmental stimuli indicate that "goal" state node ${ }_{2} \mathrm{c}_{\#}$ has been achieved rather than state note ${ }_{2} b_{1}$. Context-sensitive coding is an alternative to production systems or augmented transition networks for the representation of procedural knowledge.

\section{REFERENCES}

ANDERson, J. R. Language, memory, and thought. Hillsdale, N.J: Erlbaum, 1976.

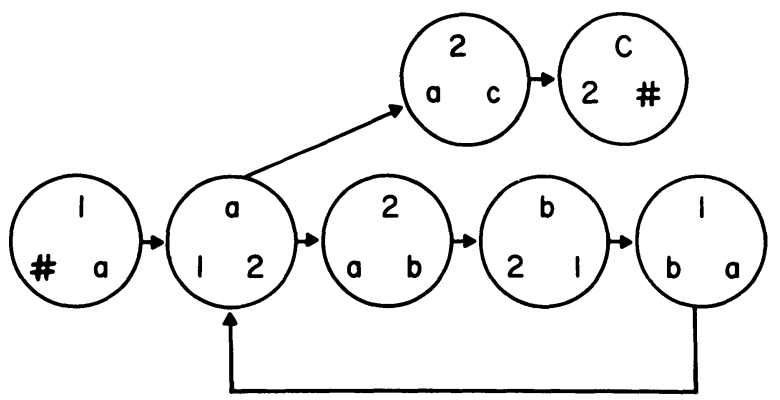

Figure 2. Context-sensitive coding of a plan that has a branching and looping structure.

LASHLEY, K. S. The problem of serial order in behavior. In L. A. Jeffress (Ed.), Cerebral mechanisms in behavior. New York: Wiley, 1951.

WICKELGREN, W. A. Context-sensitive coding, associative memory, and serial order in (speech) behavior. Psychological Review, 1969, 76, 1-15. (a)

Wickelgren, W. A. Context-sensitive coding in speech recognition, articulation and development. In K. N. Leibovic (Ed.), Information processing in the nervous system. New York: Springer-Verlag, 1969. (b)

WiCKelgren, W. A. Context-sensitive coding and serial vs. parallel processing in speech. In J. H. Gilbert (Ed.), Speech and cortical functioning. New York: Academic Press, 1972.

Wickelgren, W. A. Phonetic coding and serial order. In E. C. Carterette \& M. P. Friedman (Eds.), Handbook of perception (Vol. VII). New York: Academic Press, 1976.

\section{NOTE}

1. All of the linguistic "facts" cited in this paper were derived only slightly more systematically than is typical of linguists. I would give my two children (ages 9 and 11 years) a sentence containing nouns that was to be repeated by them with appropriate pronouns substituted for the nouns. A variety of sentence frames and orders of testing were employed, but I make no stronger empirical claim than that these "facts" are the predictions of a new context-sensitive coding theory of syntax and that I observed them to be true for my children. The purpose of this theory is to communicate that theory by means of its syntactic speech error predictions. Further empirical work is necessary to confirm the linguistic developmental facts stated in this paper. 